\title{
SpiderMAEn: recombinant spider silk-based hybrid materials for advanced energy technology
}

Heike M. Herold MSc

Doctoral candidate, Biomaterials, University of Bayreuth, Bayreuth, Germany

Tamara B. Aigner Mgr. MSC.

Doctoral candidate, Biomaterials, University of Bayreuth, Bayreuth, Germany

Carolin E. Grill Dipl. Ing.

Doctoral candidate, Biomaterials, University of Bayreuth, Bayreuth, Germany

\author{
Stefanie Krüger PhD \\ Postdoctoral Researcher, Institute of Chemistry, University of Potsdam, \\ Potsdam, Germany \\ Andreas Taubert PhD \\ Professor, Institute of Chemistry, University of Potsdam, Potsdam, Germany \\ (Orcid:0000-0002-9329-0072) \\ Thomas Scheibel PhD \\ Full Professor, Biomaterials, University of Bayreuth, Bayreuth, Germany \\ (corresponding author: thomas.scheibel@uni-bayreuth.de) \\ (Orcid:0000-0002-0457-2423)
}

A growing energy demand requires new and preferably renewable energy sources. The infinite availability of solar radiation makes its conversion into storable and transportable energy forms attractive for research as well as for the industry. One promising example of a transportable fuel is hydrogen $\left(\mathrm{H}_{2}\right)$, making research into eco-friendly hydrogen production meaningful. Here, a hybrid system was developed using newly designed recombinant spider silk protein variants as a template for mineralization with inorganic titanium dioxide and gold. These bioinspired organic/inorganic hybrid materials allow for hydrogen production upon light irradiation. To begin with, recombinant spider silk proteins bearing titanium dioxide and gold-binding moieties were created and processed into structured films. These films were modified with gold and titanium dioxide in order to produce a photocatalyst. Subsequent testing revealed hydrogen production as a result of light-induced hydrolysis of water. Therefore, the novel setup presented here provides access to a new principle of generating advanced hybrid materials for sustainable hydrogen production and depicts a promising platform for further studies on photocatalytic production of hydrogen, the most promising future fuel.

\section{Introduction}

The limited availability of fossil fuels such as petroleum, gas or coal in conjunction with the growing energy demand worldwide boosts the search for alternative, environmentally friendly energy sources. The extraction of non-renewable fuels is limited to confined areas; the fuels are depleted more and more and, hence, become increasingly expensive. Further, the emission of greenhouse gases (GHGs) during the burning of fuels promotes global warming. In contrast, sunlight, wind, tides and geothermal energy are generally available worldwide. However, the production of storable (liquid) fuels from renewable energy sources which is needed for example for portable applications or for transportation is still an issue. ${ }^{1,2}$ While various biofuels (ethanol, biodiesel etc.) exist, molecular hydrogen $\left(\mathrm{H}_{2}\right)$ is among the most attractive fuels, since it is not based on food sources, can be stored as a liquid at low temperatures or as compressed gas and has a high energy content and, at the same time, low density and weight. Among other fuels, hydrogen can be used in eco-friendly fuel cells to produce electricity with water as the sole by-product. ${ }^{1,3}$ If used for transportation, this zeroemission fuel can reduce the worldwide effects on environmental pollution and climate change and may contribute further to better air quality of, for example, big cities with a high traffic volume.

Current hydrogen production technologies still largely rely on steam reforming, catalytic reforming or water electrolysis. ${ }^{3,4}$ Steam reforming and catalytic reforming produce hydrogen from natural carbohydrate fuels, mainly natural gas or oil, and require high energy input to start the endothermic reaction which releases hydrogen, finally leading to the emission of GHGs such as carbon monoxide $(\mathrm{CO})$ and carbon dioxide $\left(\mathrm{CO}_{2}\right)$ as by-products. Thus, fossil fuels are currently the main energy source, and the resulting hydrogen comes with a huge carbon dioxide footprint. While hydrogen production through water electrolysis relies on water as the hydrogen donor, a relatively inexpensive and widely available resource, the energy required for decomposing water into its components hydrogen and oxygen by electric current is quite high. Nevertheless, many countries have road maps for the development of technologies to gain elementary hydrogen by water splitting, ${ }^{5}$ including programs focusing on complementary use of surplus energy that is available at certain times. For example, power-to-gas concepts propose to use otherwise unused electricity for electrochemical water splitting. The resulting hydrogen could then be used again in various forms of energy-transforming devices such as gas turbines or fuel cells. ${ }^{6}$ Alternatively, hydrogen could be used for methanol synthesis (which would then be used as fuel) or for chemical transformations in, for example, metallurgy. ${ }^{7}$

Strikingly, semiconductors such as titanium dioxide $\left(\mathrm{TiO}_{2}\right)$ are able to split water photocatalytically on ultraviolet (UV) irradiation and produce molecular hydrogen. This approach provides a 'green' technology for producing hydrogen. On absorption of UV light, an electron is excited from the valence band to the conduction band, 
where it can reduce a proton, finally leading to molecular hydrogen formation. Metal/semiconductor hybrid materials (MSHMs) have attracted tremendous interest because they show great promise in the field of catalysis, such as water splitting. ${ }^{8-15}$ Established protocols focus on the precipitation of semiconductor nanoparticles (NPs) such as titanium dioxide and the deposition of randomly arranged metal NPs on these existing primary particles..$^{12,14,15}$ For titanium dioxide particles, hybrids with gold $(\mathrm{Au})$ are of interest in particular since the strong localization of plasmonic near fields close to the titanium dioxide/gold interface influences optical transitions involving localized electronic states, which finally results in a higher catalytic efficiency of these nanocomposites. Recent approaches have shown that it is also possible to synthesize sideby-side morphologies called 'Janus' NPs - for example, titanium dioxide/gold particles. ${ }^{16}$ Janus particles are asymmetric particles with titanium dioxide and gold particles attached side by side with the properties of both components combined. ${ }^{17-20}$ Compared to core/shell particles, these particles show an improved performance in photocatalytic hydrogen production. ${ }^{16}$

A major disadvantage of these nanoparticulate photocatalysts is the fact that they are difficult to recycle. For long-term applications, however, easy recyclability is an important requirement, and photocatalysts that can more easily be recycled and are suited for continuous processes are necessary. Hence, photocatalysts with, for example, a film structure are interesting candidates for large-scale applications and various film-based systems of plasmonic photocatalysts have been utilized for hydrogen generation. For example, macroscopic objects such as titanium dioxide nanosheet films doped with silver $(\mathrm{Ag}) \mathrm{NPs}$ or dense films of titanium dioxide nanograss decorated with gold NPs have been reported for photocatalytic water splitting. ${ }^{21,22}$ Alternatively, instead of producing a film-like structure made of the semiconductor itself, larger objects with photocatalytic activity can be obtained by combining the actual photocatalyst with a suitable scaffold, thereby saving excess need of photocatalytic material. A good scaffold should be mechanically and chemically stable, flexible and cheap, and it should enable the synthesis of materials with a good accessibility of the photocatalyst. For example, Goutailler et al. $^{23}$ demonstrated the deposition of titanium dioxide NPs on cellulose from tetrabutylammonium bromide $\left(\mathrm{N}\left({ }^{\mathrm{n}} \mathrm{Bu}\right)_{4} \mathrm{Br}\right) /$ titanium tetraisopropoxide $\left(\mathrm{Ti}\left(\mathrm{OPr}^{\mathrm{i}}\right)_{4}\right)$ solutions in hexane. The NPs are a mixture of anatase and brookite and strongly adhere to the cellulose fibers; the cellulose/ titanium dioxide hybrids are thus mechanically rather stable and can be retrieved and washed before reuse. Daoud and $\mathrm{Xin}^{24}$ used sol-gel chemistry to coat cotton fibers with $20 \mathrm{~nm}$ anatase particles; again, the resulting materials are stable against washing and can be reused. Finally, Krüger et al. ${ }^{25}$ reported that Bombyx mori silk is a suitable scaffold for the synthesis of silk/titanium dioxide/gold hybrid materials that are efficient photocatalysts for water splitting. Overall, these examples demonstrate that the combination of a suitable scaffold with titanium dioxide-based inorganic photocatalysts could be a suitable approach toward large-scale recyclable catalysts for water splitting.
One of the issues of all the aforementioned approaches, however, is the fact that the arrangement of the titanium dioxide and the metal NPs is rather random and very difficult to control during material synthesis. This aspect should be improved in future generations of such materials. For example, as illustrated by Seh et al., ${ }^{16}$ asymmetric titanium dioxide/gold architectures are highly desirable for improved photocatalytic performance. None of the materials just described as being macroscopic and recyclable offers the possibility to precisely pre-determine the positions of the titanium dioxide and the gold components in the system. To optimize a photocatalyst further, this would be, however, an interesting pathway: if the position of all inorganic components is predetermined by the underlying scaffold, the materials could be optimized to exhibit the best possible response to the incoming electromagnetic radiation (i.e. sunlight), which would then translate into highly efficient water splitting by these highly organized catalysts.

Therefore, hybrid materials providing, for example, an organic matrix for immobilization of inorganic materials (bioinspired organic/inorganic hybrid materials) or MSHMs represent a promising approach. Such hybrid materials are arguably among the most intensely researched topics in modern materials science. Despite this, the rational and controlled formation of complex, hierarchical and multifunctional (organic/inorganic hybrid) materials remains a major challenge. The 'molecular chemist' is nowadays able to draw from a large synthetic toolbox to make complex organic, metal/organic and inorganic molecules and complexes; in contrast, the materials chemist essentially still relies on trial and error for the design of a specific material. For example, the basic principles governing the formation of metallic NPs are now understood, ${ }^{26-31}$ but even here the prediction of particle sizes, shapes, defects and so on is still in its infancy, even though the field has made significant progress in the past few years. Hybrid materials, however, have numerous advantages over purely inorganic or purely organic materials. For example, their mechanical or chemical stability can be much higher, and they may exhibit multifunctionality through incorporation of different functional components such as, for example, magnetic or photoactive inorganic and mechanically robust organic components. $^{32-38}$

As a result, the current paper describes the first steps toward such highly organized materials by making use of recombinant spider silk proteins and materials thereof as an organic template for the formation of titanium dioxide/gold hybrid materials. As a basic organic construct, the negatively charged engineered Araneus diadematus fibroin 4 with 16 repetitions of a C-module (eADF4 (C16)) and the positively charged equivalent eADF4(К16) were used. $^{39,40}$ The major advantages of this approach are that $(a)$ the silk proteins can be engineered to exhibit specific binding domains and $(b)$ the scaffolds can be processed in such a way that these binding domains are preorganized in a well-defined fashion prior to depositing gold and titanium. First, a gold-binding peptide and a titanium dioxide-binding peptide were introduced to 
Table 1. Peptide tags used in this study and their amino acid sequences and nucleotide sequences of oligonucleotides used for cloning

\begin{tabular}{|c|c|c|}
\hline Name & Peptide sequence & Nucleotide sequence \\
\hline \multirow[t]{2}{*}{$\mathrm{TiO}_{2}-\mathrm{bp}$} & QPYLFATDSLIK & $\begin{array}{l}\mathrm{TiO}_{2} \text {-fwd: CATGGGCGGCAGCGGTCAGCCGTATTTATTTGCGACCGATTCGTTAATTAAAAGCCC } \\
\text { GGGCTAATGAA }\end{array}$ \\
\hline & & $\begin{array}{l}\mathrm{TiO}_{2} \text { rev: AGCTTTCATTAGCCCGGGCTTTTAATTAACGAATCGGTCGCAAATAAATACGGCTGACCGCTG } \\
\text { CCGCC }\end{array}$ \\
\hline \multirow[t]{2}{*}{ Au-bp } & WQVQVEVQVEVQVQVQV & $\begin{array}{l}\text { Au-fwd: CATGGGCGGCAGCGGTTGGCAGGTTCAGGTGGAAGTTCAGGTGGAAGTGCAAGTTCAGGTGC } \\
\text { AAGTGAGCCCGGGCTAATGAA }\end{array}$ \\
\hline & & $\begin{array}{l}\text { Au-rev: AGCTTTCATTAGCCCGGGCTCACTTGCACCTGAACTTGCACTTCCACCTGAACTTCCACCTGAA } \\
\text { CCTGCCAACCGCTGCCGCC }\end{array}$ \\
\hline ntag Cys & GCGGSGGGGSGGGG & Analogous cloning strategy described previously $y^{39,41}$ \\
\hline
\end{tabular}

silk at the genetic level. The expectation was to synthesize a highly organized hybrid material where titanium and gold domains alternate but are in intimate contact with each other to produce a highly efficient water-splitting catalyst with a side-byside architecture, but on a much larger scale than individual Janus NPs as previously published. ${ }^{16}$

\section{Experimental section}

\subsection{Materials}

All chemicals and consumables were purchased from SigmaAldrich (St Louis, Missouri), Carl Roth (Karlsruhe, Germany) or VWR (Dresden, Germany) at per analysis quality, if not stated otherwise.

\subsection{Genetic modification of spider silk variants eADF4 (C16) and eADF4(k16)}

The cloning cassette developed by Huemmerich et al. ${ }^{39}$ for the construction of synthetic spider silk genes allows the attachment of nucleotide sequences to the $5^{\prime}$ or $3^{\prime}$ end of silk genes, leading to amino- or carboxyl-terminal tags of the proteins. Briefly, $\mathrm{TiO}_{2}$-bp- and Au-bp-encoding oligonucleotide pairs (forward and reverse) were designed to enable hybridization of the single-stranded deoxyribonucleic acid (DNA) to form a double-stranded DNA comprising sticky ends which resemble the restriction pattern of the restriction enzymes NcoI and HindIII (shown in Table 1). Following digestion of the pCS vector backbone with the same endonucleases, the respective tagencoding sequences could be ligated with the $\mathrm{pCS}$ cloning vector. Concerning the amino terminal $n \operatorname{tag}^{\mathrm{Cys}}$, this sequence was used previously, ${ }^{42}$ and, thus, the sequence was already available in a pCS vector. Enzymatic cleavage and subsequent subcloning into a silk-gene-containing vector generated the desired hybrid sequences. Further subcloning of these sequences into a pET expression vector using the restriction enzymes $N c o$ I and HindIII allowed the isopropyl $\beta$-D-1-thiogalactopyranoside (IPTG)inducible gene expression in Escherichia coli. The produced constructs can be seen in Figure 1.

\subsection{Protein production}

All cloned silk constructs were produced as described previously. ${ }^{39}$ Briefly, the expression vectors containing the modified silk constructs were transformed into E. coli BL21-Gold (DE3) (Novagen). Transformed bacterial cells were subsequently cultured in a lysogeny broth medium in a 1.3 liter Minifors reactor (Infors HT, Bottmingen, Switzerland). Fermentation was performed at high cell density and gene expression was induced at an optical density at $600 \mathrm{~nm}\left(\mathrm{OD}_{600}\right)$ of 45 using IPTG at a final concentration of $1 \mathrm{mM}$. Cell pellets were harvested after $4 \mathrm{~h}$. For constructs comprising a T7-tag, heat step purification was applied as shown previously. ${ }^{39}$ Since proteins were produced in soluble form, bacterial cells were lysed by addition of lysozyme and subsequent ultrasonication. After removal of cell debris in a centrifugation step, a heat denaturation step was used for all nonHis-tagged variants to induce precipitation of contaminating E. coli proteins, while the silk proteins stayed soluble. The soluble silk proteins in the supernatant were salted out by ammonium sulfate precipitation, and the pure silk protein was lyophilized for storage. In the case of constructs containing a $\mathrm{His}_{6}$-SUMO tag, they were column purified as described previously. ${ }^{43}$ Briefly, after protein production in E. coli BL21-Gold (DE3), the silk protein was purified using a Ni-NTA column (HisTrap FF, GE Healthcare, Little Chalfont, UK). Then, the SUMO tag was cleaved by SUMO protease, which was afterward removed again using a Ni-NTA column. The proteins were finally identified by sodium dodecyl sulfate-polyacrylamide gel electrophoresis (SDS-Page) followed by silver staining.

Figure 1. Spider silk variants developed for gold and titanium dioxide binding. Biomineralization peptides were (genetically) added $\mathrm{N}$ - or C-terminally to the sequences of eADF4(C16) or

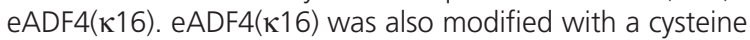
containing ntag ${ }^{\text {Cys }}$. The T7 tag was replaced by a His ${ }_{6}$-SUMO tag (SUMO, small ubiquitin-like modifier) in Au-bp-eADF4(к16) to support purification 


\subsection{Matrix-assisted laser desorption ionization-time- of-flight mass spectrometry}

The molecular weight (MW) of the proteins was analyzed by matrix-assisted laser desorption ionization-time-of-flight mass spectrometry (Maldi-TOF MS). Therefore, proteins were dissolved in $6 \mathrm{M}$ guanidinium thiocyanate and subsequently dialyzed against $20 \mathrm{mM}$ 2-amino-2-(hydroxymethyl)propane-1,3-diol (Tris)/ hydrochloric acid $(\mathrm{HCl})(\mathrm{pH} 7 \cdot 5)$. The aqueous solution was mixed with the matrix sinapinic acid $(20 \mathrm{mg} / \mathrm{ml}$ in $90 \%$ acetonitrile and $0 \cdot 2 \%$ trifluoroacetic acid) and spotted on a target plate for analysis on a Bruker Reflex III mass spectrometer (Bruker, Germany), which was equipped with a $337 \mathrm{~nm}$ nitrogen gas $\left(\mathrm{N}_{2}\right)$ laser.

\subsection{Film preparation and structuring}

Protein films were prepared on high-density polyethylene (HDPE) foil. Therefore, lyophilized ntag ${ }^{\mathrm{Cys}} \mathrm{eADF} 4(\kappa 16)$ or eADF4(C16)$\mathrm{TiO}_{2}$-bp was dissolved in formic acid and circular films spanning an area of $1 \mathrm{~cm}^{2}\left(0.5 \mathrm{mg}\right.$ protein $\left./ \mathrm{cm}^{2}\right)$ or an area of $9.1 \mathrm{~cm}^{2}(0.33 \mathrm{mg}$ protein $/ \mathrm{cm}^{2}$ ) were cast directly on the foil. The films were allowed to dry and either used directly or further modified with silk patterns as reported previously. ${ }^{44}$ Shortly, patterned polydimethylsiloxane (PDMS) stamps $\left(\sim 0.5 \times 1 \mathrm{~cm}^{2}\right.$; groove width: $50 \mu \mathrm{m}$ ridge width: $20 \mu \mathrm{m}$; height difference: $25 \mu \mathrm{m})$ were applied on dried silk films (one stamp per $1 \mathrm{~cm}^{2}$ film and six stamps per $9 \cdot 1 \mathrm{~cm}^{2}$ film). Then, $5 \mu \mathrm{l}$ of a $10 \mathrm{mg} / \mathrm{ml}$ silk solution of the second silk - that is, eADF4 (C16) $-\mathrm{TiO}_{2}$ in the case of ntag ${ }^{\mathrm{Cys}} \mathrm{eADF} 4(\kappa 16)$ silk film - was applied next to the open side of the channels. The channels filled due to capillary forces and PDMS stamps were carefully removed after drying. Film thicknesses after drying were determined using a micrometer screw (digital external micrometer, Holex, Germany).

\subsection{Gold coupling and toning}

For gold coupling, ntag ${ }^{\mathrm{Cys}} \mathrm{eADF} 4(\kappa 16)$ films were first incubated for 2-3 $\mathrm{h}$ in 3,3',3"-phosphanetriyltripropanoic acid (TCEP) buffer (1 mM TCEP, $2 \mathrm{mM}$ phosphate buffer, $15 \mathrm{mM}$ sodium chloride $(\mathrm{NaCl}), 0 \cdot 1 \mathrm{mM} \mathrm{2,2}, 2^{\prime \prime}, 2^{\prime \prime \prime}$-(ethane-1,2-diyldinitrilo)tetraacetic acid (Edta), $\mathrm{pH} 6 \cdot 5$ ) to reduce the thiol groups of the cysteine tag. A Monomaleimido Nanogold aliquot $(1.4 \mathrm{~nm}$ colloidal gold, Nanoprobes, USA) was suspended in water and incubated with the films. After $3 \mathrm{~h}$ of incubation, the films were washed three times with buffer ( $2 \mathrm{mM}$ phosphate buffer, $15 \mathrm{mM}$ sodium chloride, $0 \cdot 1 \mathrm{mM}$ Edta, $\mathrm{pH} 6 \cdot 5$ ), followed by a washing step with $0 \cdot 3 \%$ Tween 20 in phosphate-buffered saline and two washing steps with water. Then, gold toning was performed by first applying LI Silver solution (prepared as recommended by the user protocol, Nanoprobes, USA) for $5 \mathrm{~min}$. The films were washed twice with water before LM GoldEnhance solution (prepared as recommended by the user protocol, Nanoprobes, USA) was applied twice for $5 \mathrm{~min}$. Finally, the films were washed twice with water.

\subsection{Titanium dioxide mineralization}

Titania NPs were synthesized according to a published protocol by Goutailler et al. $^{23}$ In short, titanium tetraisopropoxide was dissolved in a $0.05 \mathrm{M}$ tetra- $n$-butylammonium bromide solution in hexane and heated at reflux temperature for $2 \mathrm{~h}$. On cooling, the white solid was centrifuged and the particles were dried at ambient temperature in a vacuum oven overnight. Deposition of the NPs on the substrate was achieved though dip coating into freshly prepared titanium NP dispersions in Milli-Q water at room temperature.

\subsection{Particle characterization}

Scanning electron microscopy (SEM) and energy-dispersive X-ray spectroscopy were performed on a Jeol JSM-6510 with a tungsten (W) filament operated at $15 \mathrm{kV}$ and equipped with an Oxford Instruments Inca $\mathrm{x}$-act detector. Dry samples were either ground and deposited as powders on a carbon glue pad followed by sputtering with carbon using a Polaron CC7650 carbon coater or, alternatively, deposited directly on the carbon glue pad using Planocarbon and sputtered with palladium (Pd)/gold using an SC7620 minisputter coater.

X-ray powder diffraction (XRD) was performed on a Panalytical Empyrean diffractometer in a $2 \theta$ range of $4-90^{\circ}$. The X-ray wavelength was $1.5408 \AA$ (copper $(\mathrm{Cu}) \mathrm{K}_{\alpha}$ ), and the step size was $0.0131^{\circ}$. Data and particle size analysis was done using the Scherrer equation and the HighScore Plus version 4.0 (4.0.0.19037) software from Panalytical B.V.

\subsection{Photocatalysis tests}

Photocatalysis experiments were done in a planar photoreactor with defined geometry consisting of a stainless-steel housing with cooling, a Teflon inlet as the reaction chamber and a $6 \mathrm{~cm}$ dia. quartz glass window. ${ }^{45}$ The irradiation area is given by the size of the solid sample being irradiated. A solar simulator (LOT Oriel QuantumDesign), which is basically a $300 \mathrm{~W}$ xenon (Xe) lamp equipped with an AM 1.5G filter, was used as light source at an intensity of about $1000 \mathrm{~W} / \mathrm{m}^{2}$. For comparison, a $300 \mathrm{~W}$ xenon lamp without the AM $1.5 \mathrm{G}$ filter was used. The lamp delivers $1000 \mathrm{~W} / \mathrm{cm}^{2}$ independent of the attached focusing optics. The only difference is that without the filter, the lamp delivers xenon light and with the filter the optical spectrum matches that of the solar spectrum.

In a typical experiment, the catalyst and a stirring bar were placed in the reactor. Then, the setup was evacuated three times and filled with argon (Ar) to remove residual oxygen. Thereafter, $53 \mathrm{ml}$ of a 2:1 mixture of water and ethanol was added under an argon stream, and the thermostat was set to $25^{\circ} \mathrm{C}$. Both water and ethanol were treated with argon for at least $10 \mathrm{~min}$ to remove dissolved oxygen before injection into to the reaction chamber. The light source was placed at a distance of $10 \mathrm{~cm}$. After reaction (usually $24 \mathrm{~h}$ ), a sample of the gas phase was analyzed in a gas chromatograph equipped with a Carboxen column (Agilent Technologies 7890A gas chromatography (GC) system with a Jas Unis injector system) to determine the amount of hydrogen.

\section{Results and discussion}

\subsection{Cloning, protein purification and analysis}

In order to enable specific titanium dioxide or gold binding, the respective silk variants were engineered first (Figure 1). For 
titanium dioxide binding, the peptide sequence QPYLFATDSLIK, which had previously been identified by biopanning using a phage display library, ${ }^{42}$ was fused to the recombinant spider silk protein eADF4(C16) by cloning the respective hybrid constructs. A nucleotide sequence encoding the short titanium dioxidebinding peptide was fused to the $3^{\prime}$ end of the silk gene, exploiting the established cloning strategy for eADF4(C16). ${ }^{39}$ After successful subcloning of the construct eADF4(C16)- $\mathrm{TiO}_{2}$-bp into a $\mathrm{pET}$ expression vector and the transformation of $E$. coli with the respective plasmid, gene expression was induced and the heterologous protein was produced within the expression host. The protein construct could be isolated from bacterial proteins by heat step purification and was further analyzed. The apparent MW as determined using SDS-Page seemed to be slightly too high, but Maldi-TOF MS confirmed the correct MW of the fusion protein (Figure 2(a)).

To enable gold binding to silk, the recombinant spider silk protein was first modified with the gold-binding peptide (Au-bp) WQVQVEVQVEVQVQVQV, which is known to reduce gold ions in aqueous solutions and to direct crystal growth. ${ }^{46}$ Since the two silk proteins $\left(\mathrm{TiO}_{2}\right.$-bp and $\mathrm{Au}$-bp modified) had to interact with each other, instead of eADF4(C16), the positively charged variant eADF4(к16) was chosen for the Au-bp modification. Previous investigations on recombinant spider silk proteins showed that the interaction between the positively and the negatively charged silk variants can be exploited to create defined, layered structures. For example, silk films or coatings could be dip coated in a solution of the oppositely charged silk variant to create a distinct, second layer of defined thickness. ${ }^{47}$ Moreover, structured films have been fabricated by casting flat films of one recombinant silk protein. By applying a PDMS stamp with a linear pattern to the film, the ridges of the second protein can be added on top of the first film to yield a patterned silk film. Again, the charge-charge interactions were of importance, since the delamination of ridges could be impeded by using oppositely charged proteins. ${ }^{44}$ On successful production, the test purifications of eADF4(К16)-Au-bp failed, and further analysis revealed that the production of the Au-bp bearing silk fusion protein lead to the formation of inclusion bodies within the bacterial cells (several variants were tested; all of them turned out to be insoluble, which would also hamper biomineralization later on; data not shown). Even Au-bp comprising silk constructs including SUMO tags (which typically increase the solubility of proteins) ${ }^{48}$ showed the formation of inclusion bodies. Since the formation of inclusion bodies occurred in all tested constructs, it seems that the periodic sequence of glutamine and valine residues within the Au-bp, which is highly prone to form beta-sheet structures, acts as a nucleus for silk aggregation into non-soluble, beta-sheet-rich structures. Since this effect could not be overcome, a different approach had to be realized to allow for controllable gold binding to the silk proteins.

The chosen second strategy to bind gold was to exploit thiol chemistry for binding of gold NPs. Thus, a silk construct containing one cysteine within a short amino terminal tag $\left(\right.$ ntag $^{\mathrm{Cys}}$ ) was created to allow for chemical coupling of surfacemodified (maleimide) gold particles. In this case, after efficient heterologous production of ntag ${ }^{\mathrm{Cys}}$-eADF4(К16) in E. coli, the protein could be purified through the established heat step purification route. Analysis of the protein's molecular weight by SDS-Page and MS confirmed the correct MW of the isolated protein (Figure 2(b)).

\subsection{Film preparation, gold coupling and gold toning}

Spider silk protein films with and without patterns were produced from $2 \%(\mathrm{w} / \mathrm{v})$ silk solutions in formic acid and cast on HDPE
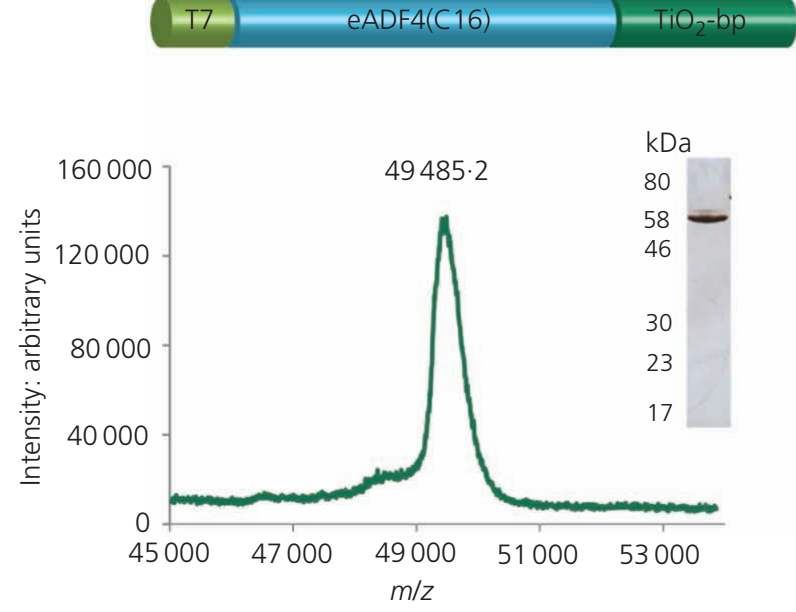

(a)

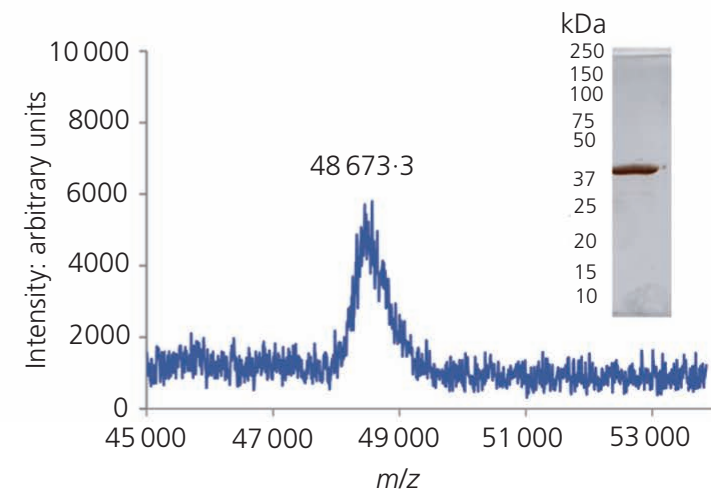

(b)

Figure 2. Analysis of the main silk variants used in this study. (a) eADF4(C16)-TiO ${ }_{2}$-bp: Maldi-TOF mass spectra (left) theoretical MW = 49518 Da; silver-stained SDS gel (right). (b) ntag ${ }^{\text {Cys }}$ eADF4( $\left.\kappa 16\right)$ : Maldi-TOF mass spectra (left) theoretical MW = 48531 Da; silver-stained SDS gel (right) 
Bioinspired, Biomimetic and Nanobiomaterials Volume 8 Issue BBN1
SpiderMAEn: recombinant spider silkbased hybrid materials for advanced energy technology

Herold, Aigner, Grill et al. foil. First, plain silk films were prepared to test and optimize gold as well as titanium dioxide binding conditions. For gold binding, thiol-bearing ntag ${ }^{\mathrm{Cys}} \mathrm{eADF} 4(\kappa 16)$ films were coupled with Monomaleimido Nanogold based on the manufacturer's protocol.
Subsequent gold toning using LI Silver solution and LM GoldEnhance yielded a gold coverage of the ground layer, as shown in Figures 3(a) and 3(b). Titanium dioxide mineralization directly on silk using titanium (IV) bis(ammonium lactato)
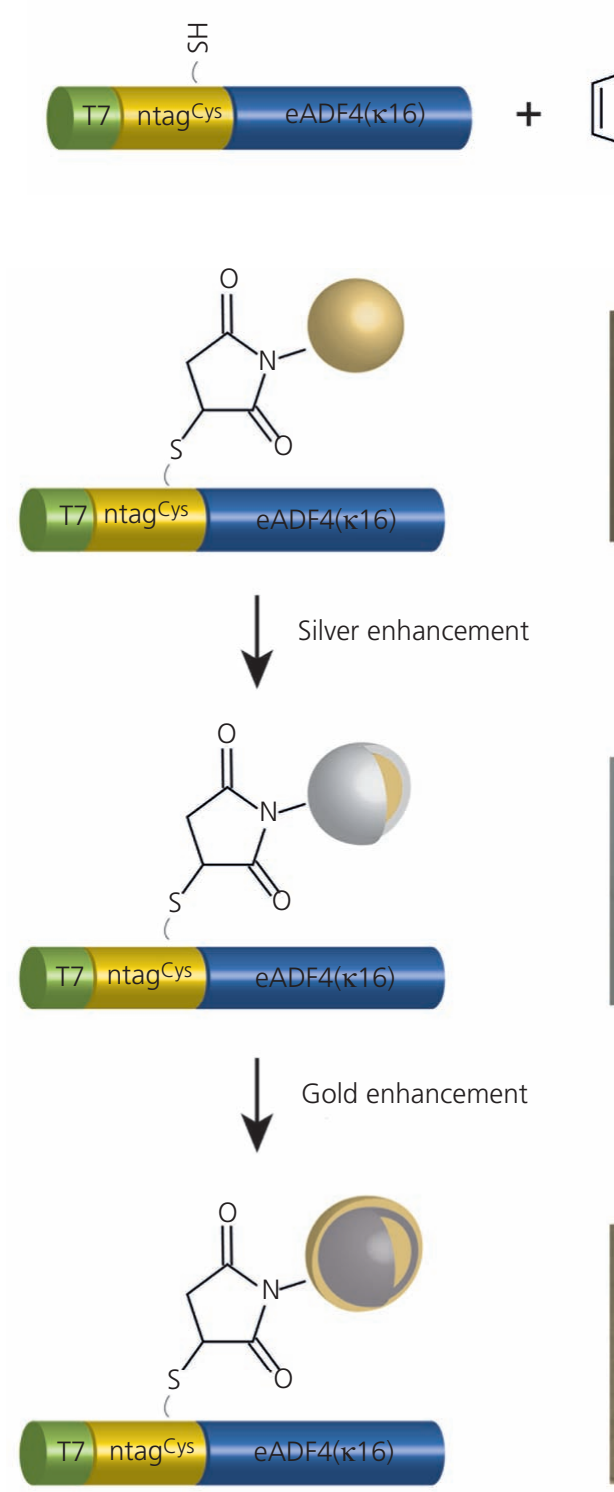

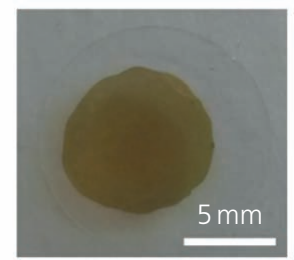

(a)
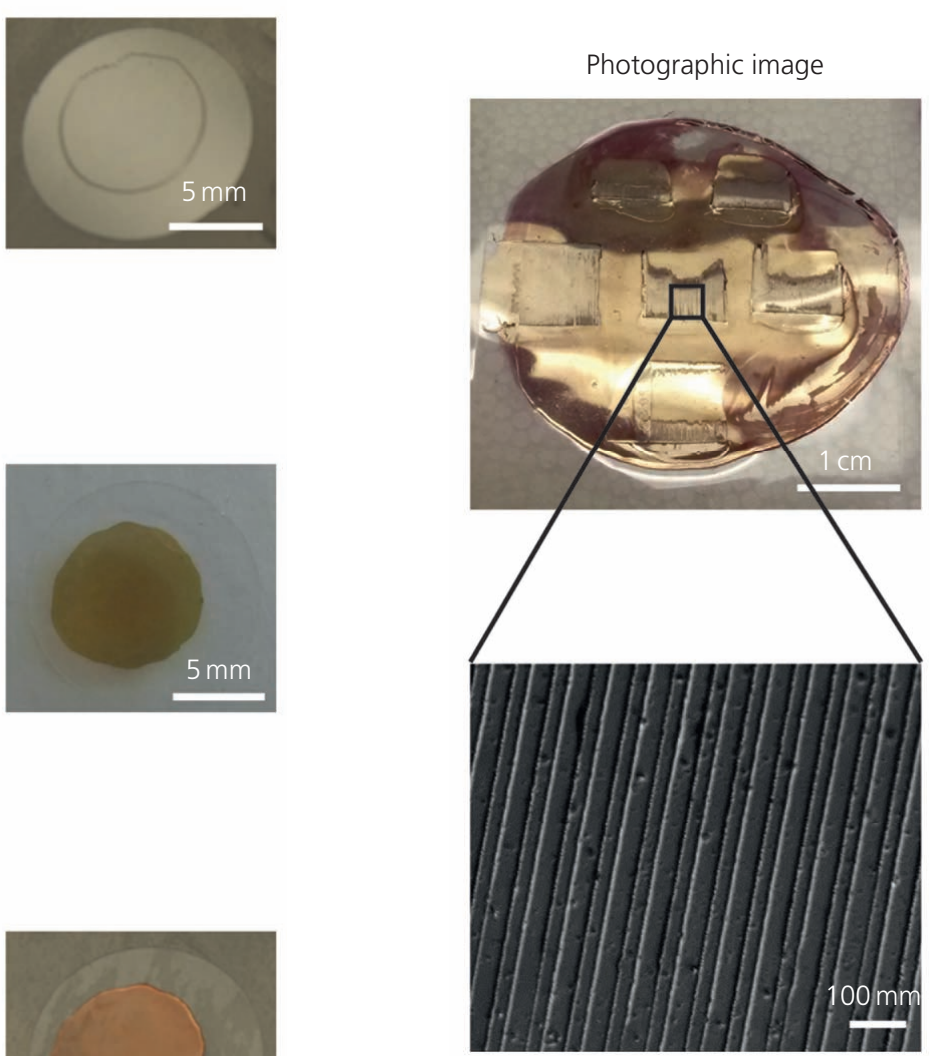

Light microscopy image

(c)

(b)

Figure 3. Gold coupling and toning of spider silk films using chemically modified gold NPs. (a) Scheme of gold coupling: the thiol- group of ntag ${ }^{\text {Cys }}$ eADF4(א16) reacts with the maleimide group of a gold NP. (b) Scheme of gold toning: after gold coupling, LI Silver solution was applied, which led to the deposition of silver around the gold NP. Then, incubation with LM GoldEnhance solution yielded a layering of gold on top of the silver. Pictures on the right are real images of the samples at each state of gold toning. (c) Photograph of the patterned film after gold coupling and toning (top) and light microscopic image of a patterned film on HDPE foil (bottom). (d) Scheme of the intended gold and titanium NP arrangement on the structured silk film - the height of the foil and film was determined with a micrometer screw and the height of the pattern was previously determined microscopically ${ }^{44}$ (the sizes of gold and titanium dioxide NPs are exaggerated for better visibility) (continued on next page) 


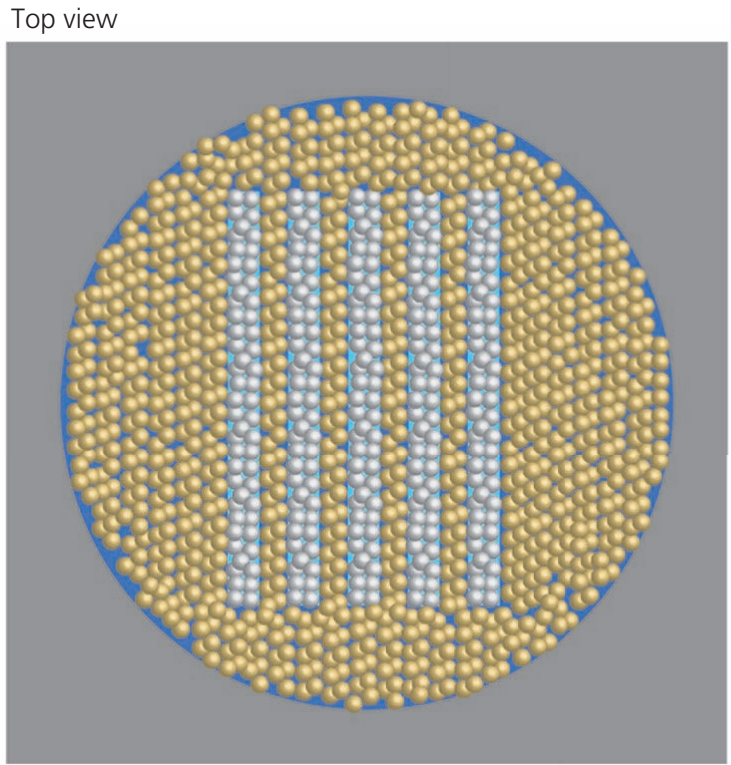

Side view

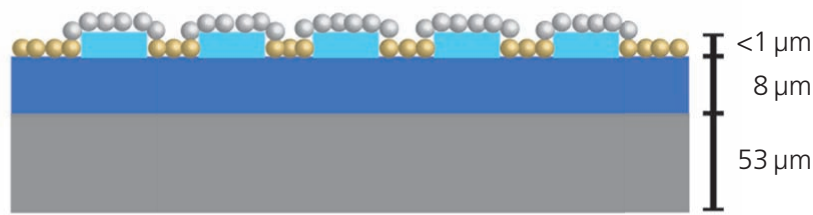

HDPE foil

ntagCyseADF4(к16)

eADF4(C16)- $\mathrm{TiO}_{2}-\mathrm{bp}$

Au NP

$\mathrm{TiO}_{2} \mathrm{NP}$

(d)

Figure 3. Continued

dihydroxide was not successful. Therefore, another approach described in the following section was used. In order to prepare a nanocomposite comprising both gold and titanium dioxide, patterned films were prepared. The positively charged ntag ${ }^{\mathrm{Cys}} \mathrm{eADF} 4(\kappa 16)$ was used to prepare the ground layer and negatively charged eADF4(C16)- $\mathrm{TiO}_{2}$-bp for the stripes. Due to the extensive washing during gold coupling and toning, some of the films tended to delaminate at the edges. However, intact (not delaminated) films could be used for further mineralization with titanium dioxide and photocatalysis experiments.

\subsection{Titanium dioxide mineralization}

For the fabrication of the hybrid materials, titanium NPs were synthesized according to existing protocols in the literature. ${ }^{23,49-53}$ The synthesis according to Goutailler et al. ${ }^{23}$ was confirmed to be the best in terms of product quality - that is, homogeneity, crystallinity and size distribution - while other synthetic approaches lead only to rather ill-defined titanium products and were thus not further considered. Figure 4 shows a representative SEM micrograph and an XRD pattern of these particles. SEM clearly showed that the samples were highly aggregated NPs (Figure 4(a)), which is not unusual for titanium NPs. However, the particle aggregates appear rather homogeneous and there is no indication of heterogeneous aggregation leading to very different aggregate shapes and types. All aggregates are rounded or roundish, and their size ranges from a few hundreds of a nanometer to $c$. $20 \mu \mathrm{m}$. There is no indication of very large aggregates of over $100 \mu \mathrm{m}$ into the millimeter dimension. Therefore, while samples showed a rather homogeneous size distribution of the primary NPs (20-50 nm range), the aggregates formed from these particles had a somewhat broad size distribution but without the presence of extraordinarily large aggregates. Overall, the SEM data thus indicate that the samples were rather homogenous, albeit aggregated. Complementary XRD pattern could be assigned to an anatase phase (Figure 4(b)), but the presence of other (minority) phases could not be ruled out completely.

The final hybrid materials were made by dip coating the silk/gold substrates into the NP suspension. The resulting materials showed a white layer indicative of titanium deposition on the substrates. Although the materials looked macroscopically homogeneous, the characterization was rather difficult: the XRD patterns of the resulting hybrid materials (layers) were very noisy, and SEM was impossible due to significant sample charging. SEM and elemental mapping nevertheless suggested that the titanium particles were distributed along the line patterns of the underlying substrate.

\subsection{Water-splitting experiments}

As detailed in the experimental part, all experiments were done at $25^{\circ} \mathrm{C}$ for $24 \mathrm{~h}$. Hydrogen gas production was evaluated through GC. Prior to investigating the hybrid materials, the individual components of the system were studied as controls. These included a HDPE foil which was used as a support for the silk films, the tape used to immobilize the samples on the metal support and spider silk without the inorganic mineral components. The samples investigated thus were metal support with HDPE, metal support with tape, and metal support with HDPE, tape and silk. The results of these experiments are summarized in Table 2. These data clearly show that the hydrogen production of these control materials was essentially zero; the small values detected 


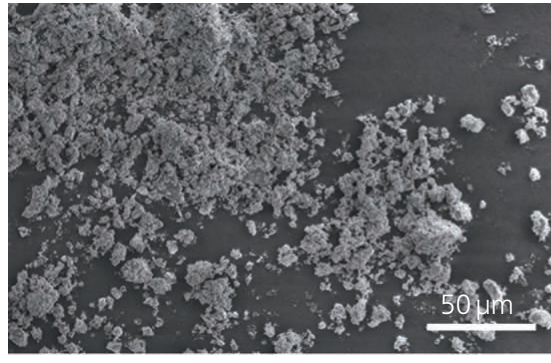

(a)

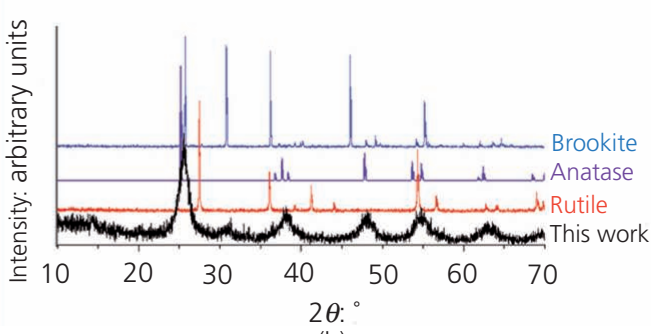

(b)

Figure 4. Titanium NPs synthesized using a protocol adapted from Goutailler et al. ${ }^{23}$ (a) SEM. (b) XRD patterns of titania made in this work using the protocol described in the experimental part are shown in black, and reference XRD patterns for single-phase titania samples (brookite, anatase, rutile) are shown to indicate the differences and matches with the XRD pattern obtained from this study's titania samples

are below the accuracy of the experimental setup. Finally, samples made from structured spider silk samples modified with Monomaleimido Nanogold gold toning (Figure 3) mounted on HDPE foil and modified with three layers of titanium particles synthesized according to Goutailler et al. ${ }^{23}$ were tested under full UV irradiation and with a $395 \mathrm{~nm}$ cutoff filter. In addition, identical samples were used where titanium was replaced with commercial Aeroxide P25 (size of single particles in the range $\sim 21 \mathrm{~nm})$.

These data clearly demonstrate that irradiation of the films modified with the gold-coupled and gold-toned film and a sufficient amount of titania did in fact catalyze the photochemical splitting of water. Consistent with the literature, the hydrogen production efficiency was higher in the absence of a cutoff filter. With the cutoff filter (eliminating the UV radiation), the hydrogen production dropped roughly by a factor of 50-100. It must be noted here that the effective values under identical conditions (383.2 against 265.6 and 16.8 against $61 \cdot 2)$ should not be overinterpreted - it may be possible that there is a larger variation in the individual experiments. This is, however, difficult to

Table 2. Hydrogen production using a xenon lamp, with or without a $395 \mathrm{~nm}$ cutoff filter

$\begin{array}{lccc}\text { Samples } & \begin{array}{c}\text { Cutoff } \\ \text { filter } \\ (395 \mathbf{~ n m})\end{array} & \begin{array}{c}\text { Hydrogen } \\ \text { fraction in } \\ \text { gas volume: } \\ \%\end{array} & \begin{array}{c}\text { Hydrogen } \\ \text { produced: } \\ \boldsymbol{\mu l}\end{array} \\ \begin{array}{l}\text { Gold coupled + enhanced } \\ \text { + Three titanium dioxide } \\ \text { layers (synthesized }\end{array} & + & 0.12 & 16.8 \\ \text { according to Goutailler } & - & 2.64 & 383.2 \\ \quad \text { et al. }{ }^{23} \text { ) } & & & \\ + \text { Three titanium dioxide } & + & 0.42 & 61.2 \\ \text { layers (Aeroxide P25) } & - & 1.83 & 265.6 \\ & & & \\ \text { Control samples } & - & 0.01 & 1.9 \\ \text { HDPE } & - & 0.00 & 0.0 \\ \text { Tape } & - & 0.02 & 2.9 \\ \text { HDPE + tape + silk film } & - & & \end{array}$

quantify because the materials did show some degradation during the reaction, and so far the contribution of these degradation phenomena is not yet understood or quantified. Additionally, the (average) distance between the gold and titania particles needs further characterization. However, as photocatalytic efficiency is observed, likely the contact is very close - otherwise there would be only very limited hydrogen production. A detailed investigation on the exact distribution and arrangement of the titania and gold building blocks in the system is, however, necessary to understand better and tune the materials toward higher performance. As a note: a comparison between this system and other photocatalysts, as described in the literature, is rather difficult, since essentially all studies use different setups and parameters, as discussed in detail in a previous study. ${ }^{25}$

\section{Conclusion}

Here the authors show for the first time that recombinant spider silk films modified with gold and titanium dioxide particles can be used for hydrogen production by photocatalytic water splitting. Several new spider silk variants were engineered with specific tags for gold and titanium binding. Two oppositely charged spider silk variants were processed into flat films as well as twodimensional structured films. Monomaleimido Nanogold was covalently coupled specifically to the thiol-carrying cysteine in ntag ${ }^{\mathrm{Cys}} \mathrm{eADF} 4(\kappa 16)$. eADF4(C16)-TiO 2 -bp could be decorated with titanium dioxide particles. The gold and titanium dioxide hybrid silk constructs were used for photocatalysis tests, and hydrogen was successfully produced. Thus, this work provides a basis for further development of recyclable hybrid materials to be used in photocatalysis.

\section{Acknowledgements}

This work was financially supported by Deutsche Forschungsgemeinschaft grant SPP1569 (SCHE603/15-1 and 15-2 to T.S., TA571/11-1 and 11-2 to A.T.) and the Bavarian Research Foundation (DOK-175-15 to T.B.A.). Further, the authors would like to thank Stephan Jokisch, Johannes Diehl and Andreas Schmidt (University of Bayreuth) as well as Michael Schwarze and Hannah Klein (Technical University of Berlin). 
Bioinspired, Biomimetic and Nanobiomaterials Volume 8 Issue BBN1
SpiderMAEn: recombinant spider silkbased hybrid materials for advanced energy technology Herold, Aigner, Grill et al.

\section{REFERENCES}

1. Rogner HH and Scott DS (2000) Building sustainable energy systems: the role of nuclear-derived hydrogen. Proceedings of the Nuclear Production of Hydrogen - First Information Exchange Meeting, Paris, France, pp. 11-24.

2. BP (2016) BP Statistical Review of World Energy June 2016. London, UK.

3. IEA (International Energy Agency) (2004) Hydrogen and Fuel Cells. OECD Publishing, Paris France.

4. Schnurnberger W, Janßen $\mathrm{H}$ and Wittstadt U (2004) Wasserspaltung mit Strom und Wärme (Stadermann G (ed.)). FVS Themen 2004, Berlin, Germany, pp. 50-59. See http://www.fvee.de/fileadmin/publikationen/ Themenhefte/th2004/th2004_03_01.pdf (accessed 19/12/2018).

5. Deutsches Institut für Normung (DIN) e. V. (2016) Deutsche Normungsroadmap Energiespeicher, Version 1. DIN e. V, Berlin, Germany (in German).

6. Koch K (2014) Hydrogen in a future mobility. Erdöl, Erdgas, Kohle 130(5): 174-187.

7. Ausfelder F, Beilmann C, Bertau M et al. (2015) Energy storage technologies as options to a secure energy supply. Chemie Ingenieur Technik 87(1-2): 17-89.

8. Kudo A and Miseki Y (2009) Heterogeneous photocatalyst materials for water splitting. Chemical Society Reviews 38(1): 253-278.

9. Ni M, Leung MKH, Leung DYC and Sumathy K (2007) A review and recent developments in photocatalytic water-splitting using $\mathrm{TiO}_{2}$ for hydrogen production. Renewable \& Sustainable Energy Reviews 11(3): 401-425.

10. Youngblood WJ, Lee SHA, Maeda K and Mallouk TE (2009) Visible light water splitting using dye-sensitized oxide semiconductors. Accounts of Chemical Research 42(12): 1966-1973.

11. Linic S, Christopher P and Ingram DB (2011) Plasmonic-metal nanostructures for efficient conversion of solar to chemical energy. Nature Materials 10(12): 911-921.

12. Zhang XM, Chen YL, Liu RS and Tsai DP (2013) Plasmonic photocatalysis. Reports on Progress in Physics 76(4): 1-41.

13. Li ZS, Luo WJ, Zhang ML, Feng JY and Zou ZG (2013)

Photoelectrochemical cells for solar hydrogen production: current state of promising photoelectrodes, methods to improve their properties, and outlook. Energy \& Environmental Science 6(2): 347-370.

14. Osterloh FE (2013) Inorganic nanostructures for photoelectrochemical and photocatalytic water splitting. Chemical Society Reviews 42(6): 2294-2320.

15. Rawalekar S and Mokari T (2013) Rational design of hybrid nanostructures for advanced photocatalysis. Advanced Energy Materials 3(1): 12-27.

16. Seh ZW, Liu SH, Low M et al. (2012) Janus $\mathrm{Au}-\mathrm{TiO}_{2}$ photocatalysts with strong localization of plasmonic near-fields for efficient visiblelight hydrogen generation. Advanced Materials 24(17): 2310-2314.

17. Walther A and Muller AHE (2008) Janus particles. Soft Matter 4(4): 663-668.

18. Du JZ and O'Reilly RK (2011) Anisotropic particles with patchy, multicompartment and Janus architectures: preparation and application. Chemical Society Reviews 40(5): 2402-2416.

19. Hu J, Zhou SX, Sun YY, Fang XS and Wu LM (2012) Fabrication, properties and applications of Janus particles. Chemical Society Reviews 41(11): 4356-4378.

20. Kaewsaneha $C$, Tangboriboonrat $P$, Polpanich D, Eissa $M$ and Elaissari $A$ (2013) Janus colloidal particles: preparation, properties, and biomedical applications. ACS Applied Materials \& Interfaces 5(6): 1857-1869.

21. Liu EZ, Kang LM, Yang YH et al. (2014) Plasmonic Ag deposited $\mathrm{TiO}_{2}$ nano-sheet film for enhanced photocatalytic hydrogen production by water splitting. Nanotechnology 25(16): 1-10.

22. Liu EZ, Fan J, Hu XY et al. (2015) A facile strategy to fabricate plasmonic $\mathrm{Au} / \mathrm{TiO}_{2}$ nano-grass films with overlapping visible light- harvesting structures for $\mathrm{H}_{2}$ production from water. Journal of Materials Science 50(5): 2298-2305.

23. Goutailler G, Guillard C, Daniele S and Hubert-Pfalzgraf LG (2003) Low temperature and aqueous sol-gel deposit of photocatalytic active nanoparticulate $\mathrm{TiO}_{2}$. Journal of Materials Chemistry 13(2): $342-346$.

24. Daoud WA and Xin JH (2004) Low temperature sol-gel processed photocatalytic titania coating. Journal of Sol-Gel Science and Technology 29(1): 25-29.

25. Krüger S, Schwarze M, Baumannm O et al. (2018) Bombyx mori silk/ titania/gold hybrid materials for photocatalytic water splitting: combining renewable raw materials with clean fuels. Beilstein Journal of Nanotechnology 9: 187-204.

26. Lohse SE and Murphy CJ (2013) The quest for shape control: a history of gold nanorod synthesis. Chemistry of Materials 25(8): 1250-1261.

27. Quan ZW, Wang YX and Fang JY (2013) Correction to High-index faceted noble metal nanocrystals. Accounts of Chemical Research 46(4): 1050 .

28. Mondloch JE, Bayram E and Finke RG (2012) A review of the kinetics and mechanisms of formation of supported-nanoparticle heterogeneous catalysts. Journal of Molecular Catalysis a-Chemical 355: $1-38$.

29. Murphy CJ, San TK, Gole AM et al. (2005) Anisotropic metal nanoparticles: synthesis, assembly, and optical applications. Journal of Physical Chemistry B 109(29): 13857-13870.

30. Xia YN, Xiong YJ, Lim B and Skrabalak SE (2009) Shape-controlled synthesis of metal nanocrystals: simple chemistry meets complex physics? Angewandte Chemie-International Edition 48(1): 60-103.

31. Tao AR, Habas S and Yang PD (2008) Shape control of colloidal metal nanocrystals. Small 4(3): 310-325.

32. Judeinstein P and Sanchez C (1996) Hybrid organic-inorganic materials: a land of multi-disciplinarity. Journal of Materials Chemistry 6(4): 511-525.

33. Giannelis EP (1996) Polymer layered silicate nanocomposites. Advanced Materials 8(1): 29-35.

34. Stein A, Melde BJ and Schroden RC (2000) Hybrid inorganic-organic mesoporous silicates - nanoscopic reactors coming of age. Advanced Materials 12(19): 1403-1419.

35. Alexandre M and Dubois P (2000) Polymer-layered silicate nanocomposites: preparation, properties and uses of a new class of materials. Materials Science \& Engineering R: Reports 28(1-2): $1-63$.

36. Sanchez C, Julian B, Belleville P and Popall M (2005) Applications of hybrid organic-inorganic nanocomposites. Journal of Materials Chemistry 15(35-36): 3559-3592.

37. Le Bideau J, Viau L and Vioux A (2011) Ionogels, ionic liquid based hybrid materials. Chemical Society Reviews 40(2): 907-925.

38. Bleek K and Taubert A (2013) Errata to: New developments in polymer-controlled, bio-inspired calcium phosphate mineralization from aqueous solution [Acta Biomaterialia 9 (2013) 6283-6321]. Acta Biomaterialia 9(9): 8466

39. Huemmerich D, Helsen CW, Quedzuweit S et al. (2004) Primary structure elements of spider dragline silks and their contribution to protein solubility. Biochemistry 43(42): 13604-13612.

40. Petzold J, Aigner TB, Touska F et al. (2017) Surface features of recombinant spider silk protein eADF4(К16)-made materials are wellsuited for cardiac tissue engineering. Advanced Functional Materials 27(36): 1701427

41. Spiess K, Wohlrab S and Scheibel T (2010) Structural characterization and functionalization of engineered spider silk films. Soft Matter 6(17): 4168-4174.

42. Puddu V, Slocik JM, Naik RR and Perry CC (2013) Titania binding peptides as templates in the biomimetic synthesis of stable titania nanosols: insight into the role of buffers in peptide-mediated mineralization. Langmuir 29(30): 9464-9472. 
Bioinspired, Biomimetic and Nanobiomaterials Volume 8 Issue BBN1
SpiderMAEn: recombinant spider silkbased hybrid materials for advanced energy technology

Herold, Aigner, Grill et al.
43. Bauer J, Schaal D, Eisoldt L et al. (2016) Acidic residues control the dimerization of the N-terminal domain of black widow spiders' major ampullate spidroin 1. Scientific Reports 6: 1-9.

44. Bauer F, Wohlrab S and Scheibel T (2013) Controllable cell adhesion, growth and orientation on layered silk protein films. Biomaterials Science 1(12): 1244-1249.

45. Schwarze M, Stellmach D, Schröder M et al. (2013) Quantification of photocatalytic hydrogen evolution. Physical Chemistry Chemical Physics 15: 3466.

46. Leon L, Su W, Matsui H and Tu R (2011) Interfacial templating of inorganic nanostructures using a growth directing and reducing peptide. Soft Matter 7(21): 10285-10290.

47. Borkner CB, Wohlrab S, Möller E, Lang G and Scheibel T (2016) Surface modification of polymeric biomaterials using recombinant spider silk proteins. ACS Biomaterials Science \& Engineering 3: 767-775.

48. Marblestone JG, Edavettal SC, Lim Y et al. (2006) Comparison of SUMO fusion technology with traditional gene fusion systems: enhanced expression and solubility with SUMO. Protein Science 15(1): 182-189.
49. Mantion A and Taubert A (2007) $\mathrm{TiO}_{2}$ sphere-tube-fiber transition induced by oligovaline concentration variation. Macromolecular Bioscience 7(2): 208-217.

50. Rettenmaier J (2002) Synthese von TiO2-Nanopartikeln und Carbazol-Farbstoffderivaten für optische Anwendungen in anorganisch-organischen Hybridpolymeren. Würzburg: Bayerische Julius-Maximilians-Universität Würzburg.

51. Choi SK, Kim S, Lim SK and Park H (2010) Photocatalytic comparison of $\mathrm{TiO}_{2}$ nanoparticles and electrospun $\mathrm{TiO}_{2}$ nanofibers: effects of mesoporosity and interparticle charge transfer. Journal of Physical Chemistry C 114(39): 16475-16480.

52. Aminian MK, Taghavinia N, Iraji-zad A et al. (2006) Highly porous $\mathrm{TiO}_{2}$ nanofibres with a fractal structure. Nanotechnology 17(2) $520-525$.

53. Kaper H, Sallard S, Djerdj I, Antonietti M and Smarsly BM (2010) Toward a low-temperature sol-gel synthesis of $\mathrm{TiO}_{2}(\mathrm{~B})$ using mixtures of surfactants and ionic liquids. Chemistry of Materials 22(11): 3502-3510.

\section{How can you contribute?}

To discuss this paper, please submit up to 500 words to the journal office at journals@ice.org.uk. Your contribution will be forwarded to the author(s) for a reply and, if considered appropriate by the editor-in-chief, it will be published as a discussion in a future issue of the journal.

ICE Science journals rely entirely on contributions from the field of materials science and engineering. Information about how to submit your paper online is available at www.icevirtuallibrary.com/page/authors, where you will also find detailed author guidelines. 